\title{
Software architecture for multi-bed FDK-based reconstruction in X-ray CT scanners
}

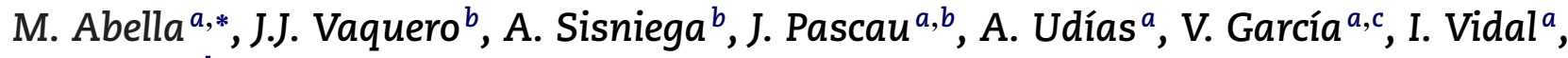 \\ M. Desco ${ }^{a, b, c}$ \\ a Unidad de Medicina Experimental. Hospital General Universitario Gregorio Marañón, Madrid, Spain \\ b Dept. Bioingeniería e Ingeniería Aeroespacial, Universidad Carlos III de Madrid, Spain \\ c Centro de Investigación en Red de Salud Mental (CIBERSAM, CIBER CB07/09/0031, Madrid, Spain
}

Keywords:

Cone-beam

FDK

$\mathrm{X}$-ray computed tomography

CT

Reconstruction

CT artifacts

A B S T R A C T

Most small-animal X-ray computed tomography (CT) scanners are based on cone-beam geometry with a flat-panel detector orbiting in a circular trajectory. Image reconstruction in these systems is usually performed by approximate methods based on the algorithm proposed by Feldkamp et al. (FDK). Besides the implementation of the reconstruction algorithm itself, in order to design a real system it is necessary to take into account numerous issues so as to obtain the best quality images from the acquired data. This work presents a comprehensive, novel software architecture for small-animal CT scanners based on cone-beam geometry with circular scanning trajectory. The proposed architecture covers all the steps from the system calibration to the volume reconstruction and conversion into Hounsfield units. It includes an efficient implementation of an FDK-based reconstruction algorithm that takes advantage of system symmetries and allows for parallel reconstruction using a multiprocessor computer. Strategies for calibration and artifact correction are discussed to justify the strategies adopted. New procedures for multi-bed misalignment, beam-hardening, and Housfield units calibration are proposed. Experiments with phantoms and real data showed the suitability of the proposed software architecture for an X-ray small animal CT based on cone-beam geometry.

\section{Introduction}

Many small animal X-ray computed tomography (CT) scanners are based on cone-beam geometry with a flat-panel detector orbiting in a circular trajectory [1-4]. This configuration presents advantages over other alternatives used in clinical and preclinical applications: reduction of acquisition time, large axial field of view (FOV) without geometrical distortions, and optimization of radiated dose [5]. Despite the existence of a remarkable progress in statistical reconstruction algorithms, approximate methods based on the algorithm proposed by Feldkamp et al. (FDK) [6] are still widely used for solving the 3D reconstruction task because of their straightforward implementation and computational efficiency [4]. Almost every aspect of the reconstruction process has been studied: there is literature on algorithm variations for different trajectories $[7,8]$, optimizations using graphic processing units (GPUs) [9-15], strategies to reduce cone beam artifacts $[16,17]$, study of consistency conditions [18], optimization of the back-projection step [19], etc. However, in a real practical system, the implementation of a reconstruction algorithm core such as FDK is just an initial step of the process, and there

\footnotetext{
* Corresponding author at: Unidad de Medicina Experimental. Hospital General Universitario Gregorio Marañón, C/Doctor Esquerdo 46, 28007 Madrid, Spain. Tel.: +34 91586 6678, fax: +34 914265108.

E-mail address: mabella@mce.hggm.es (M. Abella).
} 
are numerous additional details to consider in order to obtain good quality images at a reasonable computational cost. Many of these aspects have also been previously studied independently, for instance the characterization of the misalignments [20] or artifact corrections [21,22] but, to our best knowledge, there is no previous literature covering the complete process and detailing all the aspects to be considered: the whole workflow, the selection criteria for the different components and their right arrangement, has not been described previously. Nevertheless, when facing the design of a real scanner it is not a straightforward task to assess the optimum data workflow considering all the calibration, correction, and reconstruction issues.

This paper presents a thorough description of a novel comprehensive software architecture for tomographic image reconstruction of data acquired with small-animal CT scanners based on cone-beam geometry with circular scan trajectory. The proposed architecture covers all the steps from the system calibration and correction of the projections to the data conversion into Hounsfield units (HU), except for scatter correction. We include new procedures for multi-bed misalignment, beam-hardening, and Hounsfield units calibration. It also includes a new efficient implementation of an FDK-based reconstruction algorithm that takes advantage of system symmetries and enables a fast parallel reconstruction of different parts of the volume using a multiprocessor computer. The complete correction/reconstruction software architecture was tested on phantoms and rodent studies.

\section{Mangoose: an FDK-based CT software architecture}

The algorithm proposed by Feldkamp, Davis, and Kress, commonly referred to as FDK [6], is a well-known 3D reconstruction procedure based on an approximation of the 2D filtered backprojection (FBP) algorithm for the case when 3D data are acquired with a cone-beam geometry, e.g. flat-panel detectors and circular trajectory.

We summarize here the FDK algorithm, which is well described elsewhere. The analytical formula is derived by simply introducing a third (axial) coordinate in the FBP equation, in such a way that all the contributing rays can be considered [23]:

$f(u, v, z)=\frac{1}{2} \int_{0}^{2 \pi} W_{2} \int_{-\infty}^{\infty} R_{\beta}(s, z) h\left(\frac{S O}{S O-v}-s\right) W_{1} \partial s \partial \beta$

with

$\mathrm{W}_{1}=\frac{\mathrm{SO}}{\sqrt{\mathrm{SO}^{2}+\mathrm{z}^{2}+\mathrm{u}^{2}}}$

$\mathrm{W}_{2}=\frac{\mathrm{SO}}{(\mathrm{SO}-\mathrm{v})^{2}}$

where SO is the distance from the source to the detector, $\mathrm{z}$ is the axial coordinate, common for both detector and reconstructed volume reference frames, $s$ is the radial coordinate in the detector, and $u, v$ are the Cartesian transaxial coordinates in the reconstructed volume (Fig. 1). To facilitate geometrical calculations we make use of $u, v, z$ coordinates, common to both a virtual detector located at the FOV center and the reconstructed volume. The origin $O$ is located at the center of the FOV, which also corresponds to the central point of the virtual detector and to the center of rotation. The weighting factors $\mathrm{W}_{1}$ and $\mathrm{W}_{2}$ are introduced to compensate for the different ray lengths.

\subsection{Complete CT reconstruction software architecture}

The proposed whole CT software architecture is shown in Fig. 2. At the highest level, the CT main application, programmed in IDL 6.4 (ITT Visual Information Solutions, Boulder, $\mathrm{CO})$, includes the CT user interface and controls acquisition, calibration, and reconstruction modules. The data acquisition is performed on a Linux-based processor that controls the hardware of the CT system. The acquisition module is spawned from the CT main application following the selection by the user of the acquisition parameters, such as the X-ray energy and flux, the position of the sample, or the projection pixel size.

Projection data are pre-processed during the acquisition for dark current subtraction, flood-field correction, and recovery of dead lines in the detector (corrected by interpolation), as shown in Fig. 3. This module includes misalignment and linearization corrections, which can also be performed during acquisition. A more detailed description can be found in [5].

The proposed multi-bed reconstruction strategy is divided into two main parts: a kernel programmed in $C$ language and a graphical user interface implemented in IDL that includes modules for calibration procedures, different correction algorithms, and multi-CPU handling. The reconstruction user interface includes the VOI selection and the multi-CPU handling and is in charge of performing calls to the reconstruction kernel. The reconstruction kernel includes features to speed up the task, together with a stitching algorithm to enable large FOV reconstructions (multi-bed). Reconstruction can be performed in two modes: a fast mode that takes advantage of system symmetries but is only applicable to cylindrical VOIs symmetrical with respect to the rotation axis ('symmetrical reconstruction'), and a second (slower) mode that neither constrain the position nor the transaxial shape of the VOI ('free VOI reconstruction').

\section{Optimized FDK implementation}

The reconstruction process starts with the selection by the user of the final pixel size and the desired volume of interest, $\mathrm{VOI}_{\mathrm{T}}$, on two orthogonal projections, as shown in Fig. 4. In the case of symmetrical reconstruction, the VOI is forced to be a cylinder centered in the FOV, with the height and diameter as its only input parameters. In the case of free VOI reconstruction, the VOI is a parallelepiped, with the height and the length of the base sides defined by the geometry of selection (white lines in Fig. 4).

First of all, projection data are corrected for ring artifacts in case this feature was selected. Then, if multi-CPU feature is active, the axial dimension of $\mathrm{VOI}_{\mathrm{T}}$ is divided into as many 


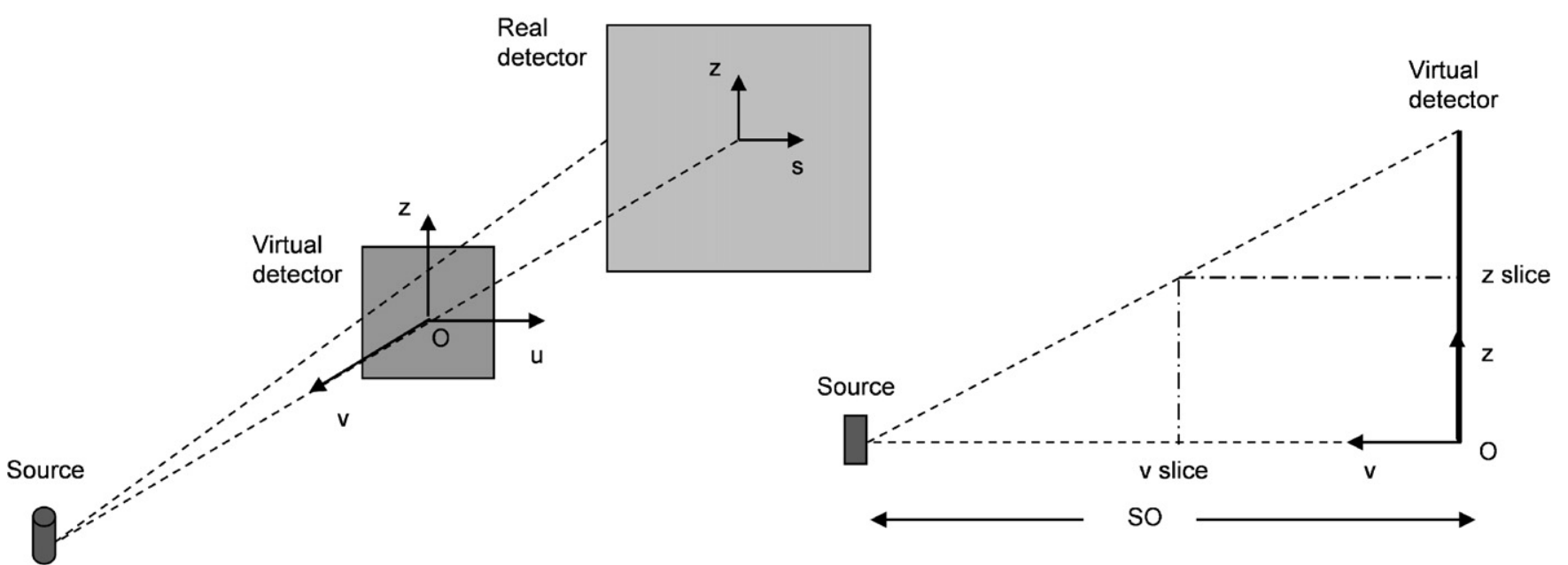

Fig. 1 - (Left) Perspective of the cone-beam geometry of the CT. (Right) Lateral view (plane $v-z$ ) from the source to the virtual detector.

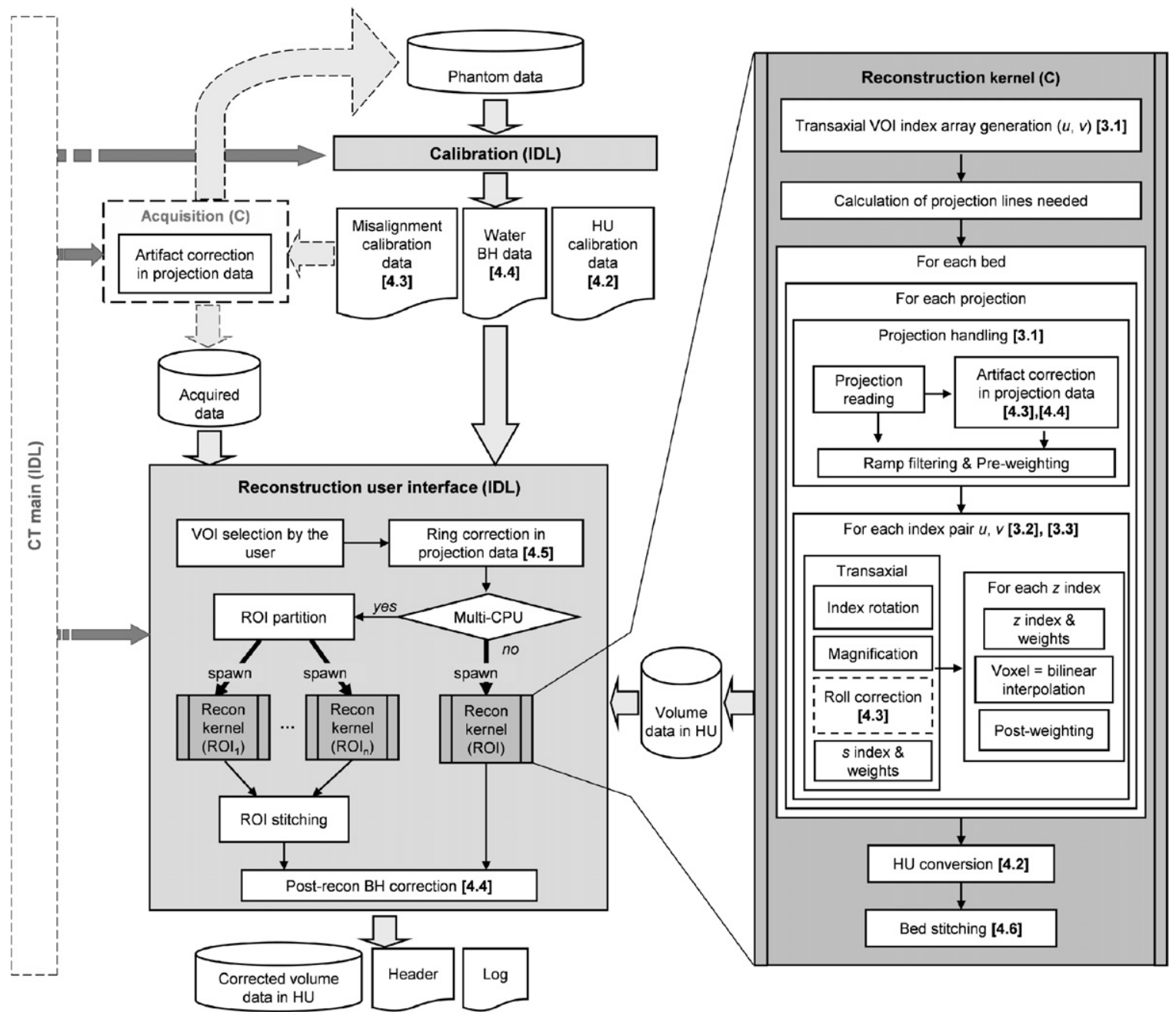

Fig. 2 - Flow-chart of the whole CT architecture for the reconstruction case without using symmetries. Elements in dotted lines are parts of the CT architecture out of the scope of this paper. Label in brackets (bold) indicate the section where that piece of the algorithm is explained. Right panel shows the details of the reconstruction kernel. 

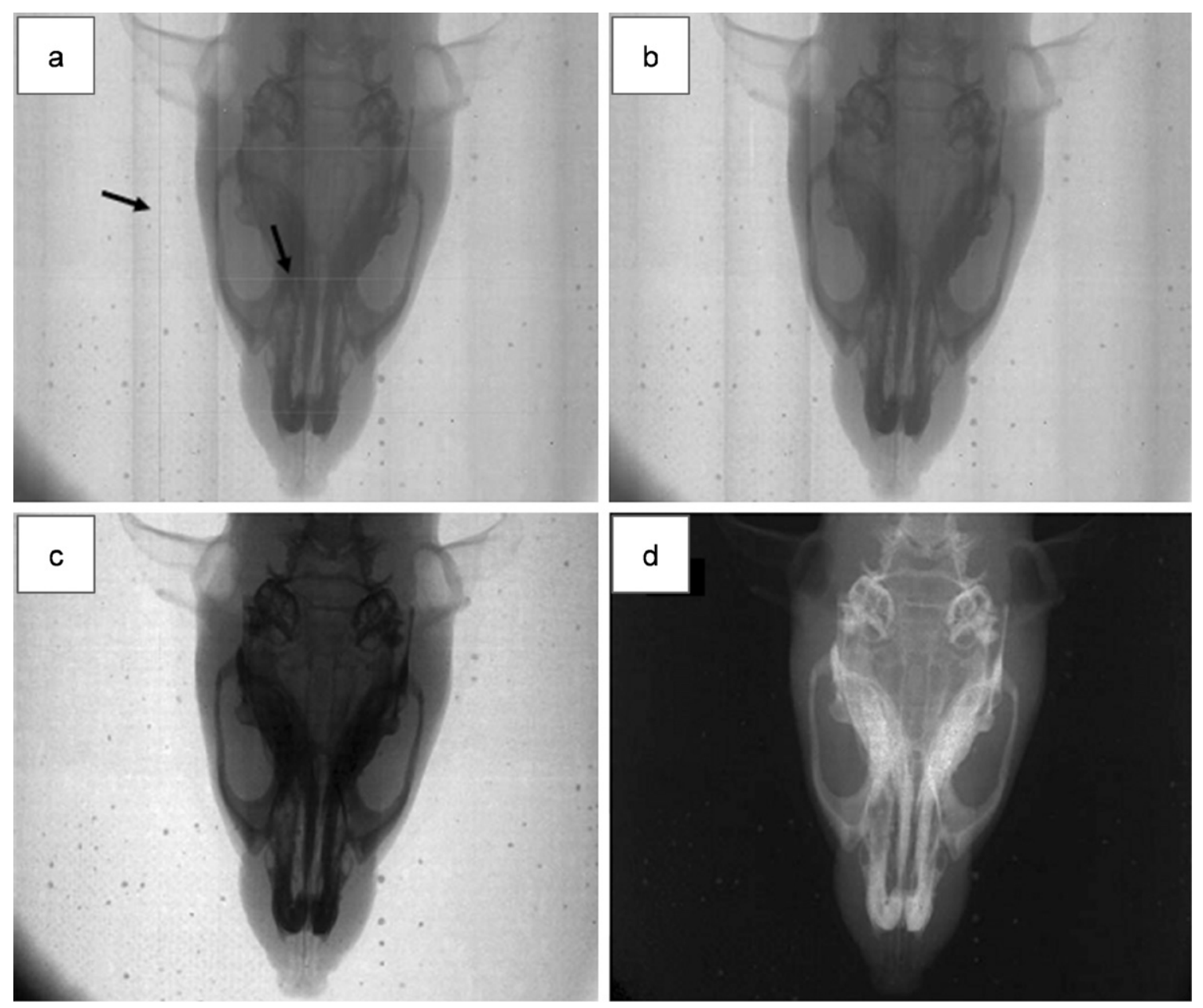

Fig. 3 - Pre-processing during acquisition. Images shown are: (a) raw data in the detector, (b) after defective lines correction (arrows in (a)), (c) after dark current and flood-field correction, and (d) after logarithmic conversion to attenuation values.

parts, $\mathrm{VOI}_{i}$, as processors are available, and an instance of the reconstruction kernel is started for each $\mathrm{VOI}_{\mathrm{i}}$.

The reconstruction kernel begins by generating an array with the indexes of the voxels inside the corresponding $\mathrm{VOI}_{\mathrm{i}}$. From this set of indexes, the algorithm calculates the subset of the projection data required to reconstruct that $\mathrm{VOI}_{\mathrm{i}}$, according to geometrical considerations. The required part of each projection is read from disk and corrected for misalignment and beam hardening (linearization), in case these features were enabled by the user and not done in the acquisition phase. Then, each projection line is ramp filtered and pre-weighted with the $\mathrm{W}_{1}$ factor, described in Eq. (2).

The back-projection step is implemented following a voxeldriven approach [24]. Each index pair $u-v$ in the index array (corresponding to a transaxial position in the VOI) is rotated by means of a rotation matrix corresponding to the projection angle, and projected to find the $s$ and $z$ indices in the projection (Fig. 1). The corresponding voxel in the reconstructed image is obtained by adding up the contributions, calculated by bi-linear interpolation in the projection data of the neighbor pixels weighted by the $\mathrm{W}_{2}$ factor described in Eq. (3).

In the case of a multi-bed VOI acquisition, a bed stitching procedure is performed after the different beds have been sequentially reconstructed and converted into Hounsfield units. Once all the instances of the reconstruction kernel are finished, the control returns to the graphical interface, where all the $\mathrm{VOI}_{\mathrm{i}}$ are stitched into the original $\mathrm{VOI}_{\mathrm{T}}$. Finally, a postreconstruction beam hardening correction is applied if the user selected this feature.

A detailed explanation of different aspects of the software is presented in the following sections.

\subsection{Projection handling}

Once the user defines the reconstruction VOI, the index pairs $u-v$ corresponding to the voxels inside the selected VOI are calculated and stored. If the selected VOI is smaller than the total physical FOV, only those projection data actually contributing to the VOI are read and pre-processed, thus reducing disk access, memory requirements, and computation time. The algorithm calculates which segments of the projection data are needed to reconstruct the user-selected VOI, according to the geometry shown in Fig. 5.

Projection data are read without truncation in the transaxial dimension to avoid artifacts in the subsequent filtering step. In the axial direction, where the limits of the area 

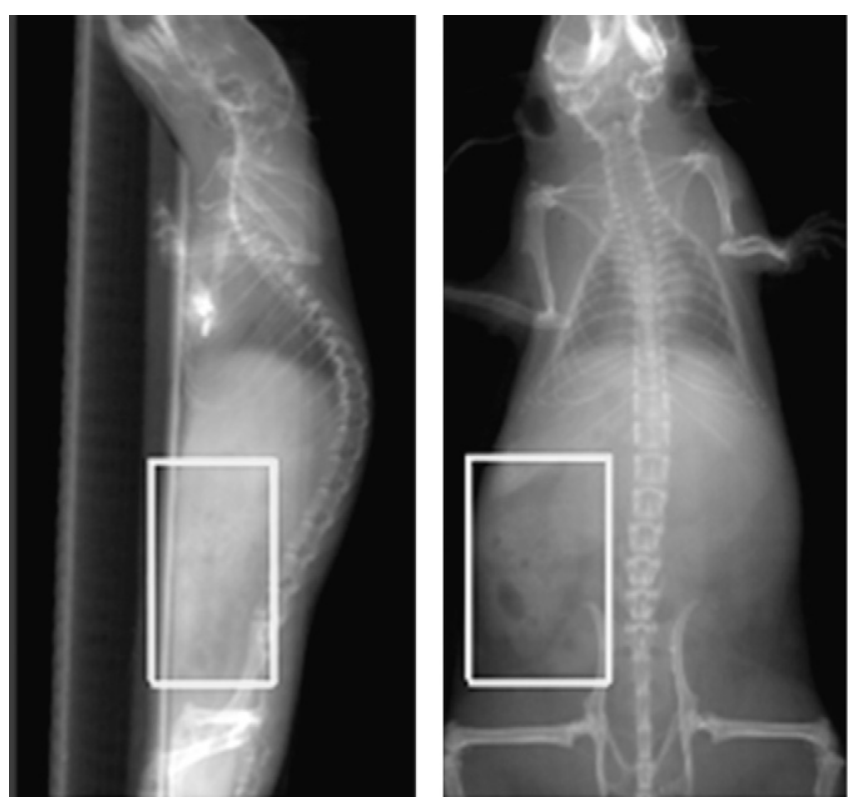

Fig. 4 - An example of sagittal (left) and coronal (right) projections used for VOI selection in the free VOI reconstruction. The white lines define the selected VOI.

required from the projections are different for each angular position, only the most restrictive limits are calculated to speed up processing. Fig. 5 shows an example of a VOI located at the top half of FOV. The upper limit ( $z_{\max }$ in Fig. 5) will correspond to the point in the upper slice of the VOI with the highest magnification (closest to the source). The lower index $\left(z_{\min }\right)$ is calculated from the projection of the point in the bottom slice of the VOI farthest from the source (minimum magnification).

\subsection{Back-projection and index rotation}

The key step in the implementation of any FBP-like algorithm is the back-projection, which consists of spreading back the filtered projection values along each ray. Ray-driven methods are generally well suited for the projection operator, but tend to introduce artifacts (Moiré patterns) in the back-projection.
Furthermore, ray-driven methods generally lead to highly inefficient non-sequential memory access patterns. For these reasons, the voxel-driven approach is the one chosen here, as it is the case in most implementations of FBP [24]. For each voxel, the cone-beam projection onto the detector is calculated and the corresponding backprojection value is computed by bi-linear interpolation of the four neighboring projection pixels. Finally, the voxel value is incremented with the result of this interpolation. The volume is generated column by column (along the $z$ coordinate) for each index pair $u-v$ in the index matrix, reusing the calculated weighting factor $\mathrm{W}_{2}$ in Eq. (3) and interpolation factors on the transaxial direction, which are independent of coordinate $z$. To optimize the implementation, the volume is stored following the order $z, x, y$, thus allowing for a sequential address of consecutive memory positions.

The tracing of rays in a rotating system can be simplified by considering that the system is static and the sample counterrotates. This leads to mathematically equivalent but simpler expressions for the rotation step. A further simplification can be done by working with a 'virtual detector', placed at the center of the FOV, which enables using a single coordinate system, common for both the projections and the FOV. Performing the rotation of the sample as a separate step and then tracing the rays requires several interpolations: one in the volume for the rotation step and another one in the projection during the back-projection step. Instead, in our case the rotation is obtained only on the volume coordinates, avoiding interpolations until the last step (back-projection), thus reducing rounding errors and saving processing time.

\subsection{Acceleration: symmetries and parallel processing}

In case of 'symmetrical reconstruction' mode, an acceleration procedure that takes advantage of the system symmetries is applied to avoid repeated calculations. Fig. 6 shows an axial view of the situation at rotation angle $\theta$. For every $\theta$, four projections 90 degrees apart are read and processed at a time ( $P 1$, P2, P3, and P4 in Fig. 6). For each voxel position within the VOI ( $u, v$ indexes corresponding to point $A$ in Fig. 6$)$, the algorithm calculates the rotated position for angle $\theta\left(u_{a}\right.$ and $v_{a}$
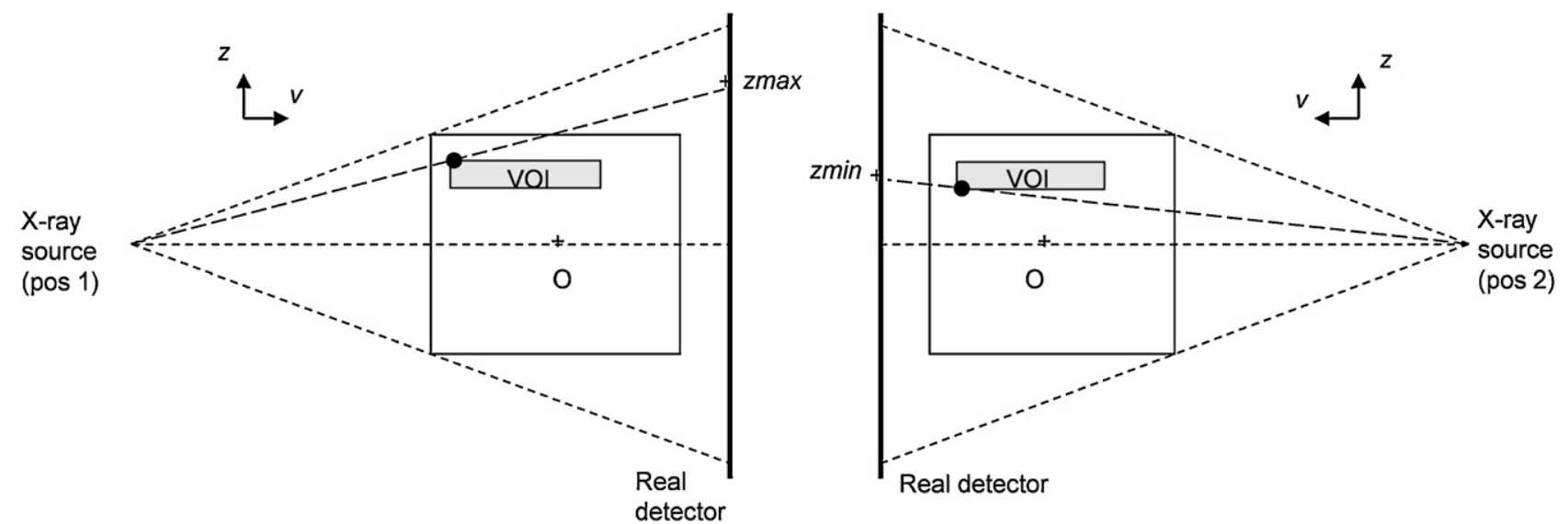

Fig. 5 - Example of projection data selection for a VOI located at the top half of the FOV. (Left) Axial view showing the projection where $z_{\max }$ value is found. (Right) Transaxial view showing the projection where $z_{\min }$ value is found. 


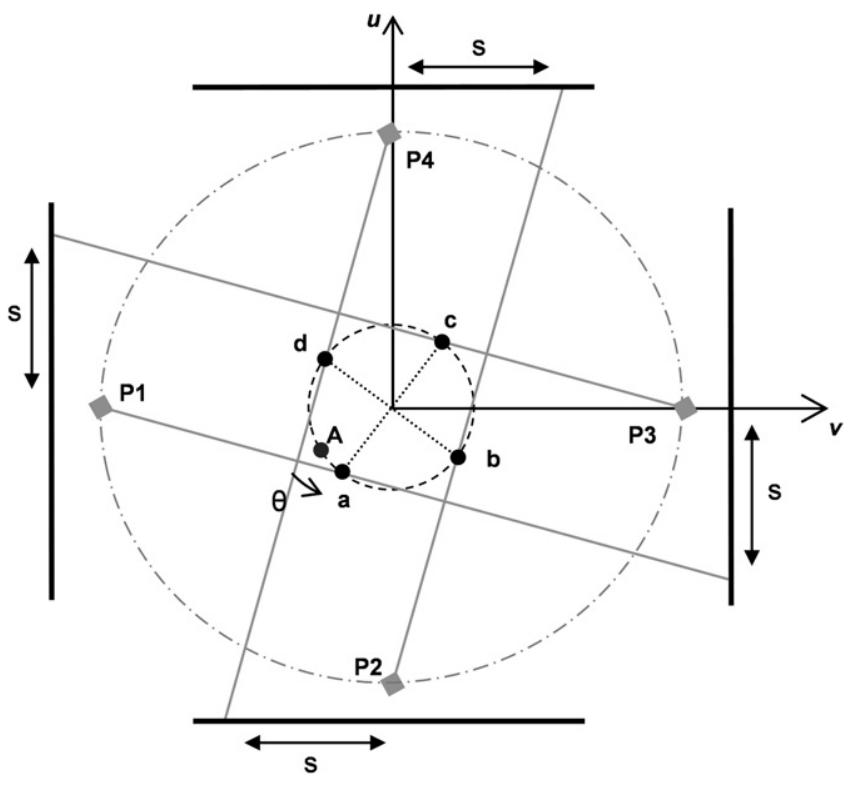

Fig. 6 - Sketch of geometrical symmetries. $u, v, s$, and z indexes calculated for point $a$ are used for four source positions 90 degrees apart (P1, P2, P3, and P4).

indexes corresponding to point $a$ in Fig. 6), and the position of its projection ( $z$ and $s$ indexes).

The same point a rotated 90,180 , and 270 degrees, corresponding to positions $b, c$, and $d$, will have the same projection position ( $z$ and $s$ indexes) and values for $u$ and $v$ indexes following the relations:

$u_{a}=\cos \theta u_{A}-\sin \theta v_{A}$

$v_{a}=\sin \theta u_{A}+\cos \theta v_{A}$

$u_{a}=v_{b}=-u_{c}=-v_{d}, v_{a}=-u_{b}=-v_{c}=u$

Another method for speeding up the process is to perform parallel processing. The algorithm was designed to be able to reconstruct slices independently, thus allowing parallel reconstruction of different parts of the VOI, that only require a straightforward stitching step.

\section{Calibration and corrections}

This section describes the different strategies selected for calibration and artifact correction.

Calibration procedures directly related to image reconstruction include obtaining the magnification value, which affects the voxel size, the Hounsfield unit conversion data, the detector misalignments, and the beam-hardening data.

Mechanical misalignment corrections in single bed acquisitions are performed on the projections as a previous step before reconstruction. For multi-bed studies, the error derived from misalignments of the bed displacement with respect to the axis of rotation is compensated during the bed-stitching step (previously estimated by means of a calibration method).
Two algorithms have been implemented for beam hardening correction: a linearization correction, an approach currently available in most commercial scanners, which assumes that all the materials in the sample have homogeneous $\mathrm{X}$ ray attenuation (similar to water), and a post-reconstruction algorithm that accounts for bone-related beam hardening.

Finally, a ring artifact correction algorithm can optionally be applied in the projection space.

\subsection{Voxel size: magnification}

Accuracy of the voxel size depends on the correct calibration of the magnification parameter and is crucial for bed stitching, volume quantification, and fusion of the CT image with other modalities. The system magnification factor can be assessed with a simple phantom acquired in two different axial positions, calculating the correspondence between a given $\mathrm{z}$ displacement (in $\mathrm{mm}$ ) monitored with the $\mathrm{z}$-axis linear motion stage and the measured number of pixels of that displacement in the reconstructed volume. The phantom used consisted in a $2 \mathrm{~mm}$ diameter metallic ball bearing embedded in the center of a low-density foam slab of dimensions $6 \mathrm{~cm} \times 6 \mathrm{~cm} \times 2 \mathrm{~cm}$ (exact dimensions are not critical).

\subsection{HU conversion}

After the reconstruction, voxel values are in arbitrary units. Conversion from these arbitrary units (commonly referred to as CT data) into standard Hounsfield units follows a linear transformation as

$H U(x)=x A+B$

where $x$ is the CT data, and A and B are parameters dependent on the scanner. To obtain these parameters we scan, at different voltage values, a phantom that consists of two cylinders of $2 \mathrm{~cm}$ diameter, one filled with water and another solid one made of polytetrafluoroethylene (PTFE). Acquisitions for each voltage are reconstructed and the mean values for air, water and PTFE (ROIs as shown in Fig. 7) are obtained. Finally, these points are fitted to a straight line, from which we obtain $A$ as the slope and $B$ as the intercept for each voltage value.

\subsection{Misalignment correction in single bed acquisitions}

Since it is almost impossible to completely remove skew and $x$-shift misalignments by mechanical calibration, a software correction of the projections before reconstruction is required. The relevant misalignments for single bed acquisitions are: tilt, roll, skew, $x$-shift, and y-shift of the detector panel (Fig. 8). The effect of these misalignments has been studied in [25]. Skew and $x$-shifts produce the most noticeable artifacts, since even small errors (about one degree or sub-pixel shifts) lead to conspicuous double edges in the image. Tilt and roll misalignments produce image elongation, but this effect is almost negligible for reasonable residual angles of misalignment after mechanical calibration (below three degrees). Effects of $y$ shifts are not clearly visible either.

Many methods for the estimation of geometrical parameters of cone-beam scanners have been proposed since 1990 


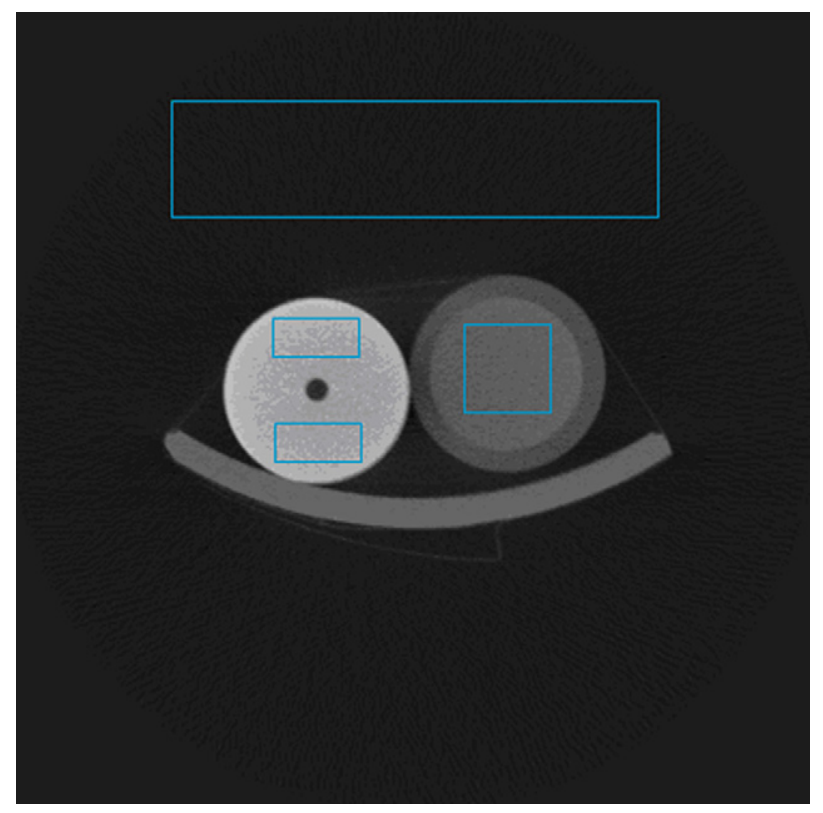

Fig. 7 - Phantom to calibrate HU with materials densities of water and PTFE.

[20,26-30]. These techniques have generally fallen into two categories: those based on iterative nonlinear optimization $[26,27]$ and those based on the direct solution of geometric equations $[20,28,29,31]$. The second type has become favored in recent years because of superior performance and ease of implementation. One important result in [26] was to show that the distance detector-object only plays the role of a magnifying factor in the reconstruction. This can be estimated a posteriori if one knows the distance between some landmarks, such as two point objects in the reconstruction, similarly to what is explained in Section 4.1 .

In [27-30], either the design of this calibration object or its positioning, or both, required very high precision. Contrarily, in $[20,32]$ the authors propose methods that avoid strict requirements on the object shape and positioning. The latter only determines the transversal shift, the skew, and, less accurately, the detector slant; other parameters, such as detector tilt, longitudinal shift, and error in the source-detector distance, cannot be determined.

The method described in [20] was selected for our architecture because it is robust, easy to implement and uses a

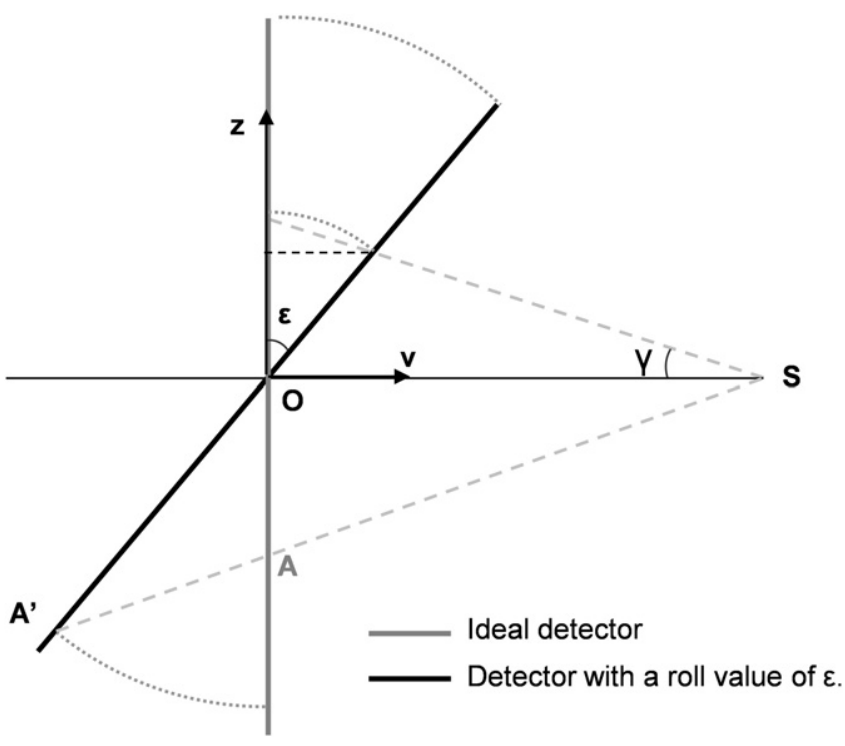

Fig. 9 - Sketch of the case when there is a roll of $\varepsilon$ degrees in the detector.

simple calibration object. Although the method is not fully general (as tilt angle is assumed to be equal zero $\theta=0$ ), it provides the set of parameters we found relevant for our scanner [25]. The measurement of these misalignments, together with the source-detector distance is performed by using a phantom consisting of two metallic bearings $0.5 \mathrm{~cm}$ in diameter embedded in a foam slab of dimensions $5 \mathrm{~cm} \times 4 \mathrm{~cm} \times 1.5 \mathrm{~cm}, 2 \mathrm{~cm}$ apart in opposite corners. Exact dimensions are not critical, as long as the ball bearings are placed one at each axial half of the FOV. The two spherical objects were considered 'point' objects whose projections trace ellipses on the detector. From the parametric description of these ellipses, the calibration geometry was analytically determined using explicit formulae.

These misalignment values are stored in a calibration file and used during the misalignment correction step (step 4.3 in Fig. 2) during either acquisition or reconstruction. Skew and linear shifts are corrected by simple image geometrical operations on the projection data.

The effect of detector roll is shown in Fig. 9, where $S$ is the X-ray source, $\varepsilon$ is the roll angle of the detector, $\gamma$ is the angle of the considered ray and the central ray of the beam, $\mathrm{A}^{\prime}$ is a pixel in the real detector, and $\mathrm{A}$ is the corresponding (a) Linear shifts

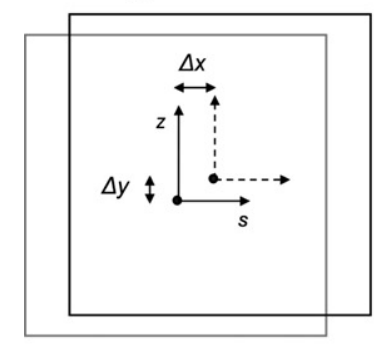

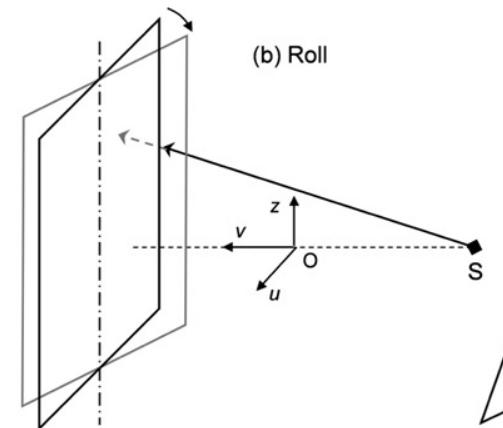
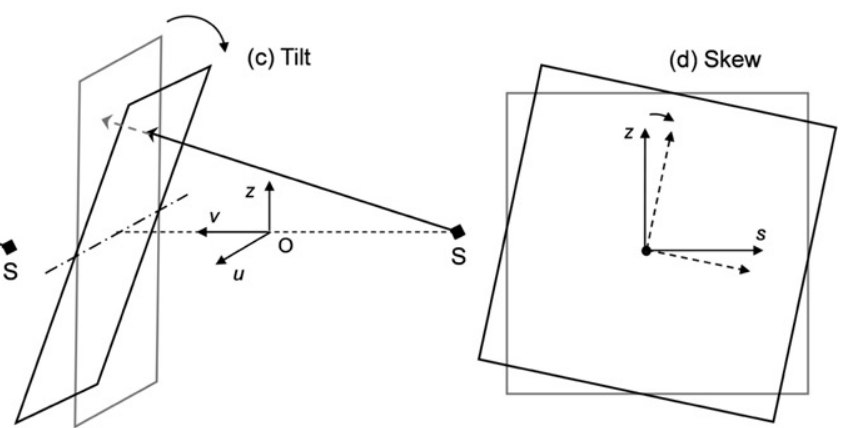

Fig. 8 - Detector misalignments. 


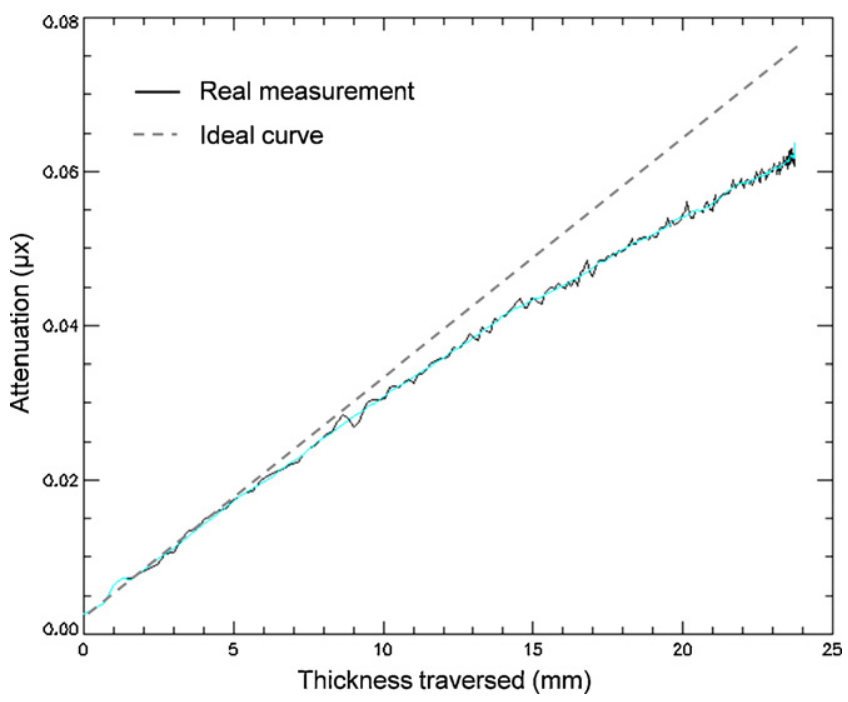

Fig. 10 - Attenuation function measured on a phantom of $24 \mathrm{~mm}$ of maximum diameter interpolated to 2400 points. The ideal attenuation function shown in grey dotted line was obtained by fitting the first $\mathbf{5 0 0}$ points in the curve to a straight line.

pixel in the ideally unrolled detector. For each point in the ideal detector we calculate the corresponding point in the real detector according to the expression:

$\left|\mathrm{OA}^{\prime}\right|=\frac{|\mathrm{OA}|}{\cos (\varepsilon)+\sin (\varepsilon) \cdot(|\mathrm{OA}| / \mathrm{SO})}$

\subsection{Beam hardening correction}

In the proposed architecture we include two beam-hardening correction algorithms. The first one is a linearization correction, which assumes that all the materials in the sample have homogeneous X-ray attenuation (similar to water), and is applied to the projections prior to reconstruction $[21,23,33]$. It is a fast correction algorithm, currently implemented in most commercial scanners, based on a linearizing function that maps polychromatic projection data obtained from the real scanner into monochromatic projection data (Fig. 10).

This function can be derived either analytically, based on the a priori knowledge of the spectrum and attenuation coefficients, or experimentally, by fitting measured data acquired from a set of samples of different thicknesses [34]. Since the X-ray spectrum is often unknown, we used a homogenous phantom made of Plexiglas (density of $1.19 \mathrm{~g} / \mathrm{cm}^{3}$ ) of semi-cylindrical shape in order to obtain a finer sampling of thickness values. To obtain the material thicknesses traversed by the different $\mathrm{X}$-rays, we made a preliminary reconstruction, segment the phantom and re-project it to obtain the thickness values actually traverse by each ray, similar to what is proposed in $[35,36]$. This approach has the advantage of neither relying on a specific phantom size or shape, nor on special phantom positioning. Finally, we obtained a profile of the attenuation associated to different thickness values. To estimate the ideal attenuation curve (corresponding to a monochromatic source), we fit the initial region of the curve

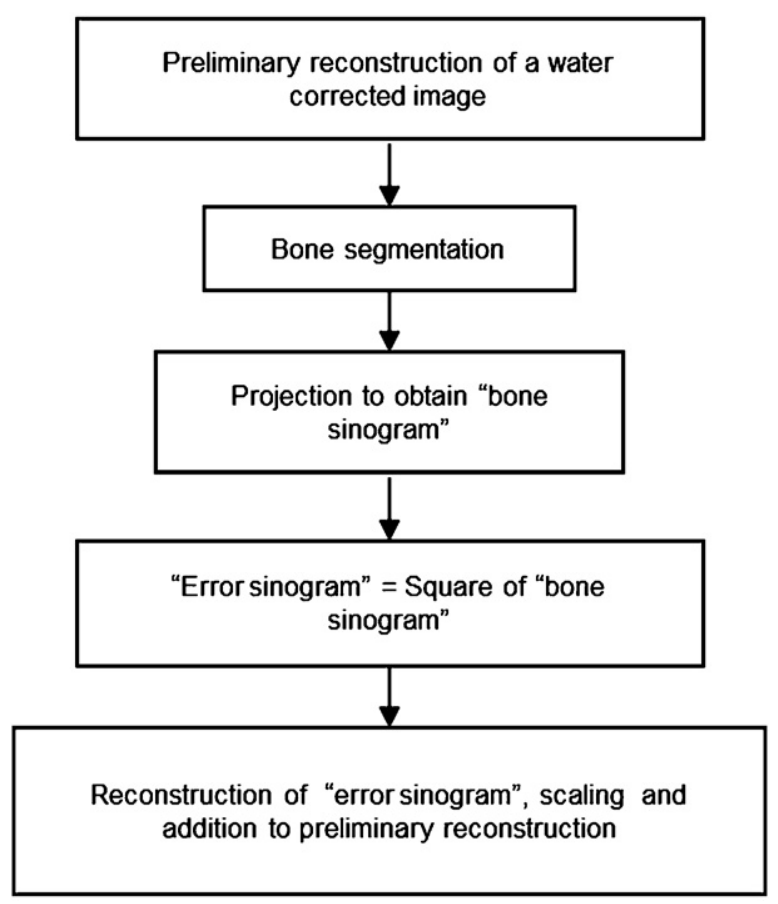

Fig. 11 - Flow-chart for the beam-hardening correction.

(small thickness), where the beam hardening effect can be considered negligible, to a linear function. Then we obtain the correction curve (from polychromatic to monochromatic equivalent beam hardening function) by fitting the curve to a polynomial of fourth grade [34]. The correction, estimated as the difference between the straight line and the polynomial fitting, is applied to projection data by means of a look-up table (step 4.4 in Fig. 2).

However, this is known to be a suboptimal correction for non-homogeneous objects, especially when they contain high density areas, such as bone. For this reason, we have also included a second algorithm which implements a postprocessing technique (step 4.4 in Fig. 2) based on the idea of Joseph and Spital [21]. This approach involves an initial FBP reconstruction and a segmentation of the bone structures, which are afterwards forward projected. This bone projection provides an estimate of the amount of nonlinear beam hardening distortion, which is then corrected for $[21,37,38]$. In our case, we use an approach similar to the one described in [37], according to the algorithm summarized in Fig. 11. The threshold for the preliminary bone segmentation is selectable by the user.

\subsection{Ring artifact correction}

In CT scanners based on flat-panel detectors and circular trajectory, images are often corrupted by ring artifacts caused by imperfections in detector elements which introduce differences in gain at specific positions in the detector array [22]. This artifact can hinder quantitative analysis in high resolution micro-CT studies; therefore, the removal or a significant reduction of these artifacts is highly desirable. 
(a)

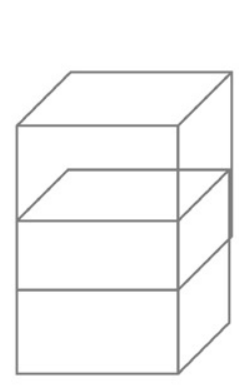

$z^{\prime} \quad z$

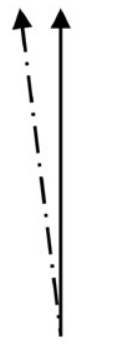

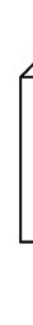

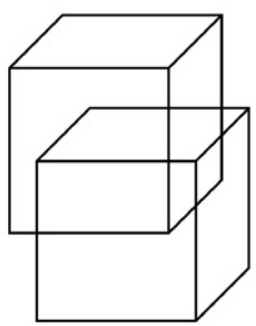

(b)

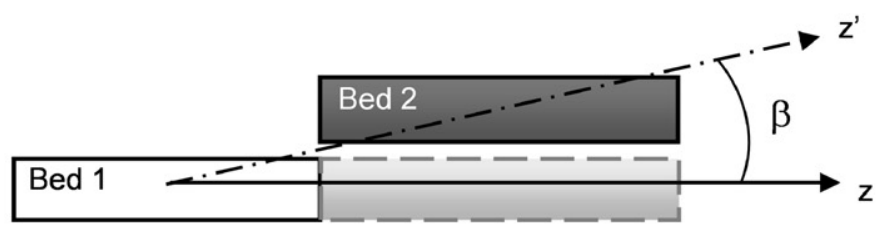

(c)

(d)
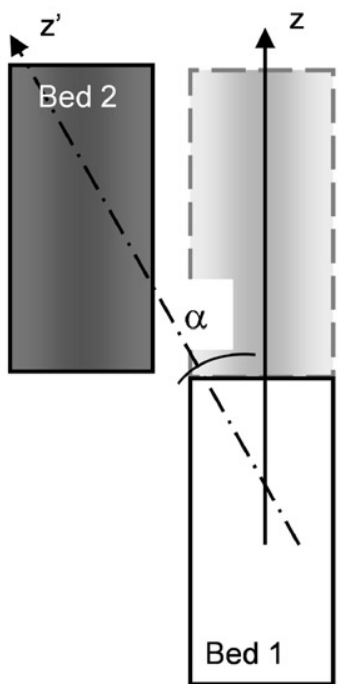

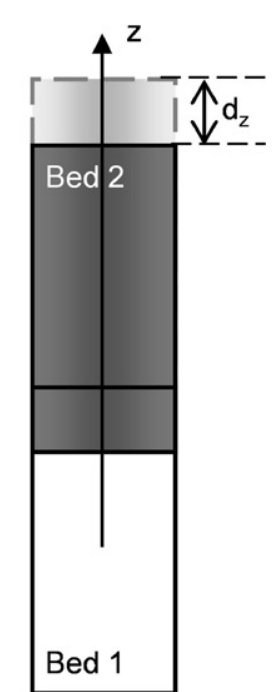

Fig. 12 - Effect of mismatch between the bed displacement axis, $z^{\prime}$, and the rotation axis, $z$. Two consecutive reconstructed FOVs with an overlap region in the case of no mismatch (a, left) and with mismatch (a, right). Sagittal (b) and coronal (c and d) views of the two bed positions. In grey, the second bed in the ideal position, and in black in the real position, for every of the three parameters that define the mismatch of the bed displacement axis and the axial axis $\left(\alpha, \beta\right.$ and $\left.d_{z}\right)$.

Our software architecture includes a method for removing the ring artifacts that survive the flat-field correction $[39,40]$. These residual ring artifacts may appear due to the no linear response of the detector elements with the incident X-ray flux, which can change between acquisitions. Among the number of methods that have been presented [22,41-50], we propose a correction algorithm that works on the projection data before reconstruction, as it can be efficiently included in the correction/reconstruction pipe-line.

We propose the following approach, following the same idea as in $[43,45,47,51]$ : we divide each oblique sinogram in parts corresponding to a subset of projection angles and correct each part independently. For each part, a low-pass version is obtained by applying a 4-pixel median filter in the radial direction (kernel size was derived experimentally and depends on the expected thickness of the ring artifacts). These lowpass versions of the oblique sinograms are subtracted from the original ones resulting in an image where ring artifacts are highlighted, appearing as straight lines along the angular direction. Finally, the correction profile is calculated as the median value along the columns (angular direction) (step 4.5 in Fig. 2).

\subsection{Bed stitching with misalignment correction}

To obtain whole body studies, a sequential multi-bed acquisition is performed by taking a series of scans. After each scan, the bed is moved to the next desired position, where the following scan is obtained with some overlap with the previous one. The bed displacement must be equal to or smaller than half of the axial FOV to prevent gaps between adjacent scans.

The most straightforward way to combine the data from multi-bed acquisitions is to reconstruct the volumes corresponding to each single axial acquisition separately and computing a weighted combination of the reconstructed slices from the different axial datasets. To minimize the effect of shading artifacts at areas far from the center slice in the overlapping regions, we apply a shading compensation technique consisting in assigning the maximum value (from the two beds) to each pixel in the overlapped area.

The bed displacement can only be mechanically aligned to the rotation axis to a certain degree and the residual misalignment introduces very conspicuous errors in bed stitching (Fig. 12(a)). We propose a new calibration method that estimates the angular misalignments between the bed movement axis and the axis of rotation ( $\alpha$ and $\beta$ in Fig. 12(b and c)) as well as the shift error in the $z$ direction $\left(d_{z}\right.$ in Fig. 12(d)).

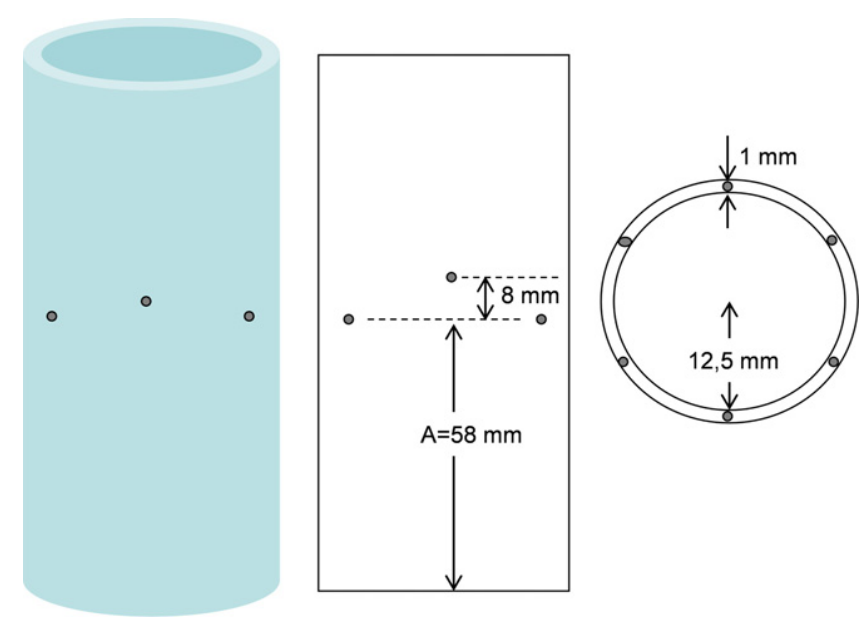

Fig. 13 - Phantom for measuring the mismatch between the bed displacement and the rotation axis. (Left) 3D view. (Right) Cross section and cylindrical projection. 

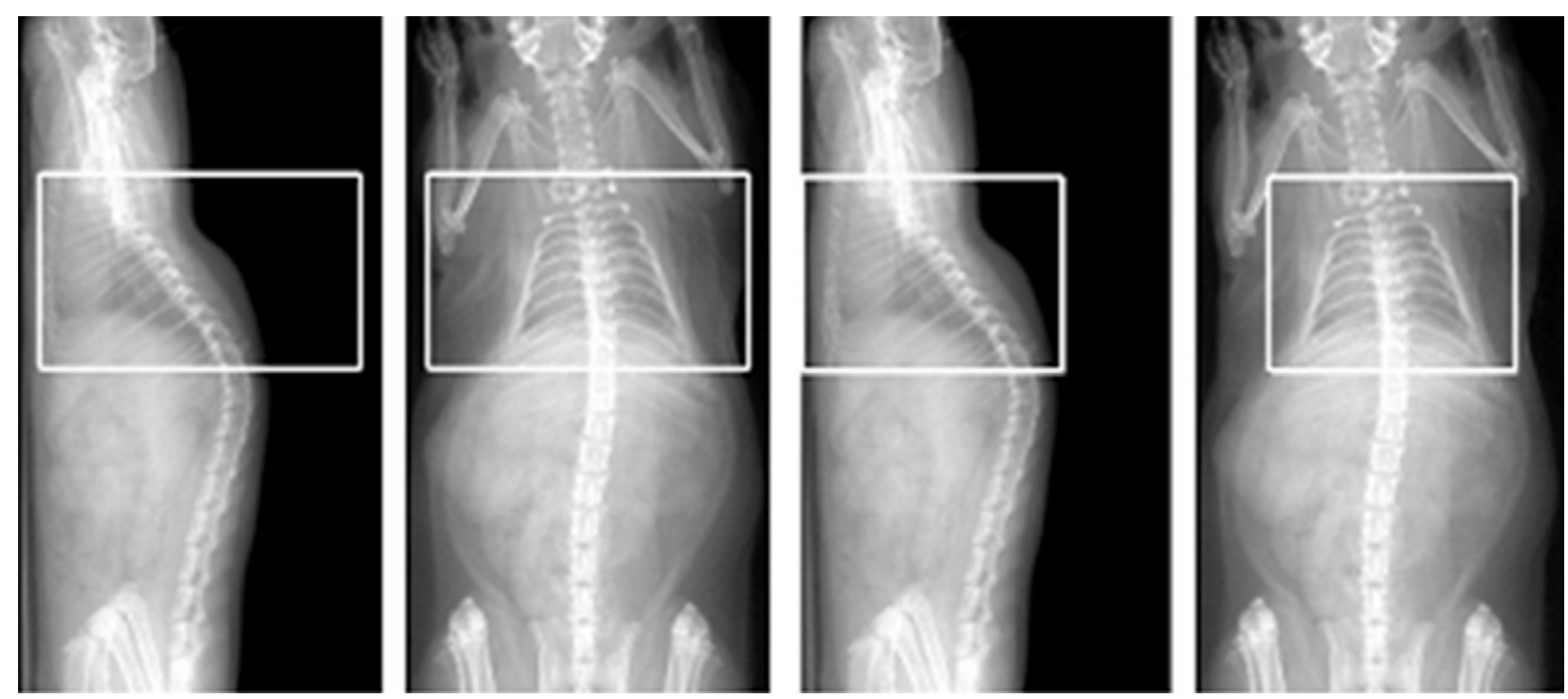

Fig. 14 - Whole-rat scout (two-beds with an overlap of $20 \mathrm{~mm}$ ) with the selection of a symmetrical (left) and a free (right) VoI adjusted to the lungs.

The calibration phantom consists of a hollow cylinder made of plexiglas with six metallic ball bearings $1 \mathrm{~mm}$ in diameter glued to its surface, equidistantly embedded at two different axial positions, $A=58 \mathrm{~mm}$ and $B=64 \mathrm{~mm}$ (Fig. 13). These axial positions were selected to ease the placement of the phantom in the FOV: the centered slice lies at the center of the overlapping area; the distance between these two axial positions should be less than the overlapping size. In our case, bed size and overlapping size were $72 \mathrm{~mm}$ and $20 \mathrm{~mm}$, respectively:

A $=$ bed_size $-\frac{\text { overlap }}{2}$

The phantom is acquired in two partially overlapped bed positions, making sure that the bearing balls fall into the overlapping area. Both bed positions are reconstructed separately and the center of mass of each bearing is calculated. From the average displacement between the ball bearing mass centers in the two beds and the known bed displacement we can easily estimate the misalignment parameters, $\alpha, \beta$ and $d_{z}$, which are stored in a calibration file. This measured misalignment is compensated during the reconstruction process in the stitching step (step 4.6 in Fig. 2).

\section{Performance assessment}

All the studies were acquired with the CT subsystem of a small-animal PET-CT scanner (ARGUS/CT, SEDECAL, Madrid) using standard acquisition protocols usually employed in experiments with small animals. This system is fully described in [5].

Detector misalignment correction was performed during acquisition. Beam hardening of first (linearization) and second order (post-reconstruction) and ring artifact corrections were applied.
The computational cost of the solution was evaluated on a $2.66 \mathrm{GHz}$ Intel Core 2 Quad with 4 GB of RAM. We illustrate the results with a rat study consisting of a two-bed acquisition with 360 projections of $530 \times 568$ pixels per bed with an overlap of $20 \mathrm{~mm}$, corresponding with 162 slices in the reconstructed image (Fig. 14).

In order to provide standard timings, we chose a $512 \times 512 \times 512$ VOI size for the full-volume reconstructions (symmetrical and free VOI) at the center of the acquired FOV (thus containing the overlap area). We also measured times for smaller transaxial dimensions to evaluate performance at different volume sizes.

To evaluate the VOI reconstruction feature, we selected three VOIs covering the lungs: one adjusted to the lungs (free VOI) and two more using symmetrical constraints in order to evaluate the trade-offs of a fitted selection vs. a larger VOI using symmetry optimization.

The performance in terms of image quality of our complete correction/reconstruction software package was assessed by visual inspection in five homogeneous cylinders and ten whole body rodent studies (multi-bed).

\section{Results}

Table 1 shows the processing times for a full-volume reconstruction of one bed, with a volume size of $512 \times 512 \times 512$ voxels, for both symmetrical and free VOI cases. The backprojection step is the one with the highest computational cost: about $80 \%$ for both cases; results show an acceleration factor of 1.8 when using the symmetries optimization.

Fig. 15 shows the processing times for different transaxial dimensions. Projection handling is the same for all the cases since the projection lines are always processed complete to avoid artifacts in the subsequent filtering step. We can see that the increased time for HU conversion for a smaller VOI is negligible when compared to the backprojection step. 
Table 1 - Processing times in seconds for the different steps in the reconstruction of a volume of $512 \times 512 \times 512$ voxels. Projection handling includes reading, filtering, weighting, and beam hardening linearization.

\begin{tabular}{lrr} 
Step & Time for symetrical VOI (s) & Time for free VOI (s) \\
\hline Projection handling & 37.1 & 37.1 \\
Backprojection & 184.7 & 349.8 \\
Bed stitching (162 slices) & 2.7 & 2.7 \\
Misalignment correction between beds (612 slices) & 9.1 & 9.0 \\
HU conversion & 6.5 & 6.5 \\
Writing to disk & 1.5 & 1.5 \\
Total & 241.6 & 400.5 \\
\hline
\end{tabular}

Table 2 - Processing times for different VOIs covering the lungs. Projection handling includes reading, filtering, weighting, and beam hardening linearization.

\begin{tabular}{llllllr} 
Type of volume & VOI size (277 slices) & Projection handling & Back-projection & Bed stitching & HU conversion & Total \\
\hline Fitted free & $356 \times 274$ & 25.1 & 125.0 & 0.8 & 1.9 & 165.2 \\
Symmetrical & $512 \times 512$ & 25.2 & 114.7 & 1.6 & 3.8 & 146.5 \\
Symmetrical & $468 \times 468$ & 25.1 & 95.7 & 1.4 & 3.1 & 126.4 \\
\hline
\end{tabular}

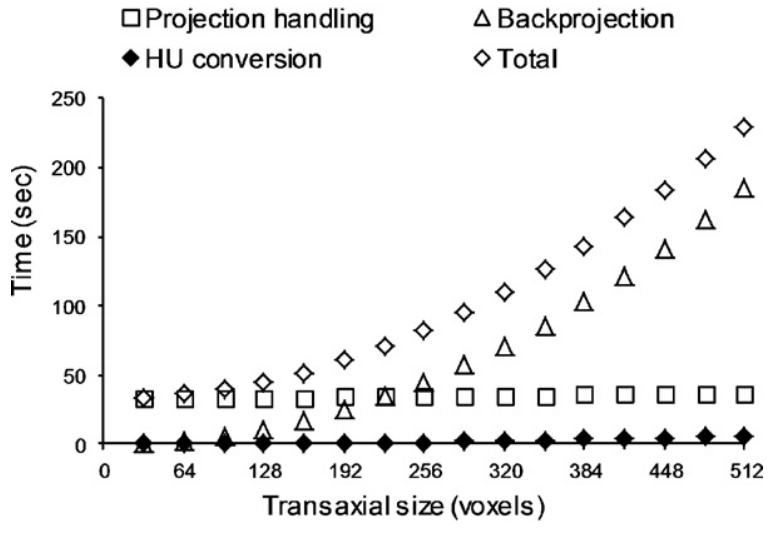

Fig. 15 - Processing times for VOIs with 512 slices and different transaxial size within one bed.

Fig. 16 shows the analysis for multi-CPU, showing that the time for the handling of multiple VOIs can be considered negligible.

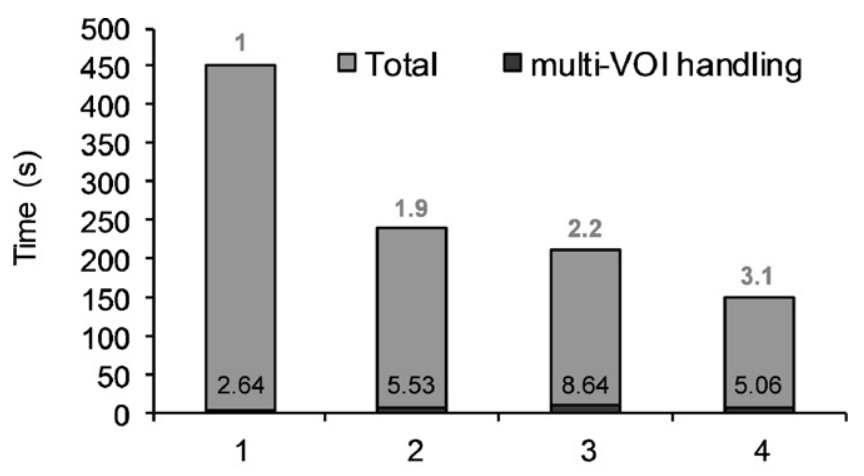

Number of CPUs in use

Fig. 16 - Reconstruction times of the complete rat study (two beds) using 1, 2, 3 or 4 CPUs. Black numbers on top of dark bars show the corresponding value (multi-VOI handling). Numbers in grey font on top of light grey bars show the acceleration factor for each case.
Table 2 shows the results when selecting a VOI covering the lungs. We can see that sometimes it can be better to reconstruct a larger VOI if it allows for the use of symmetry optimization.

Fig. 17 shows the results of the beam-hardening correction algorithm based on linearization on a homogeneous cylinder.

Fig. 18 shows the removal of the streak artifacts obtained with the post-processing correction. Finally, we show images from three rodent studies to evaluate the overall performance of the reconstruction algorithm. Note the absence of artifacts due to detector misalignments, rings, and beam hardening, as well as the satisfactory result of the stitching procedure.

Fig. 19 presents a single-bed rodent study with contrast media (0.4-ml injection of Iopamiro 300), and Fig. 20 presents the render of a multi-bed rodent study.

\section{Discussion}

In this work we have presented a comprehensive, novel software architecture to implement the complete reconstruction process for small-animal CT scanners based on cone-beam geometry with circular scanning trajectory. The proposed architecture covers all the steps from the system calibration to the volume reconstruction and conversion into Hounsfield units. We include an efficient implementation of an FDK-based reconstruction algorithm that takes advantage of system symmetries and allows for parallel reconstruction using a multiprocessor computer. We obtain an acceleration factor of 1.8 when using the symmetries optimization. A further optimization of the back-projection step could be done using the distance-driven technique described in [52], whose authors claim to be suitable for both projection and backprojection, offering low arithmetic cost and highly sequential memory access pattern, while reducing artifacts. A common strategy to speed up the back-projection step involves the use of graphics processing units (GPUs). In recent years several implementations have resulted in processing time reduction factors of up to 40 [9-15], most of them using the compute unified device architecture (CUDA). The modularity in our 

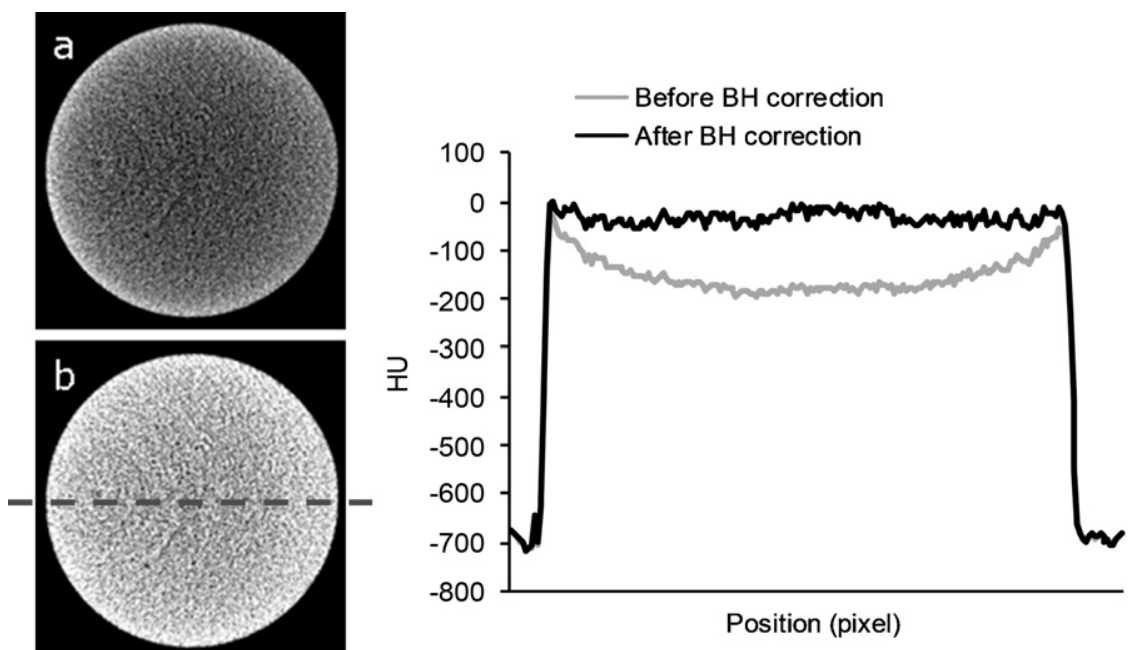

Fig. 17 - Cupping artifact produced by water-related beam hardening, before and after correction in a low dose acquisition of a cylinder. (Left) View of a transaxial slice with the grey scale range adjusted to highlight the cupping effect before (a) and after (b) the correction. (Right) Profile along the doted line.
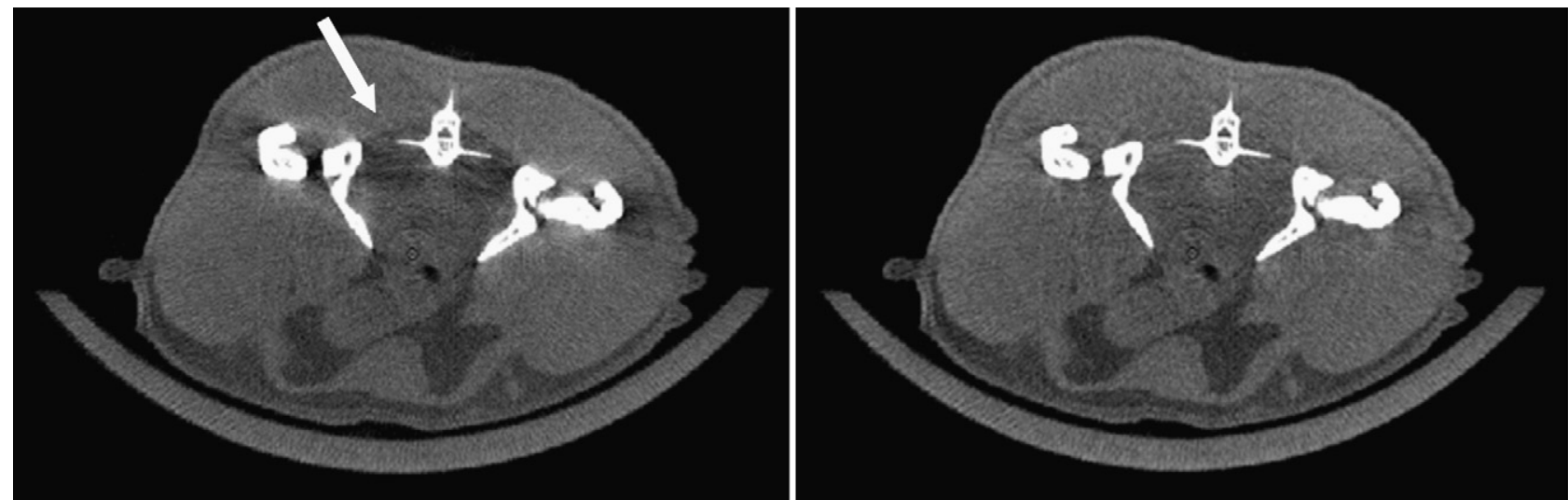

Fig. 18 - Beam hardening artifacts in a mouse study before (left) and after correction (right). The white arrow shows the dark streaks that are removed with the correction.
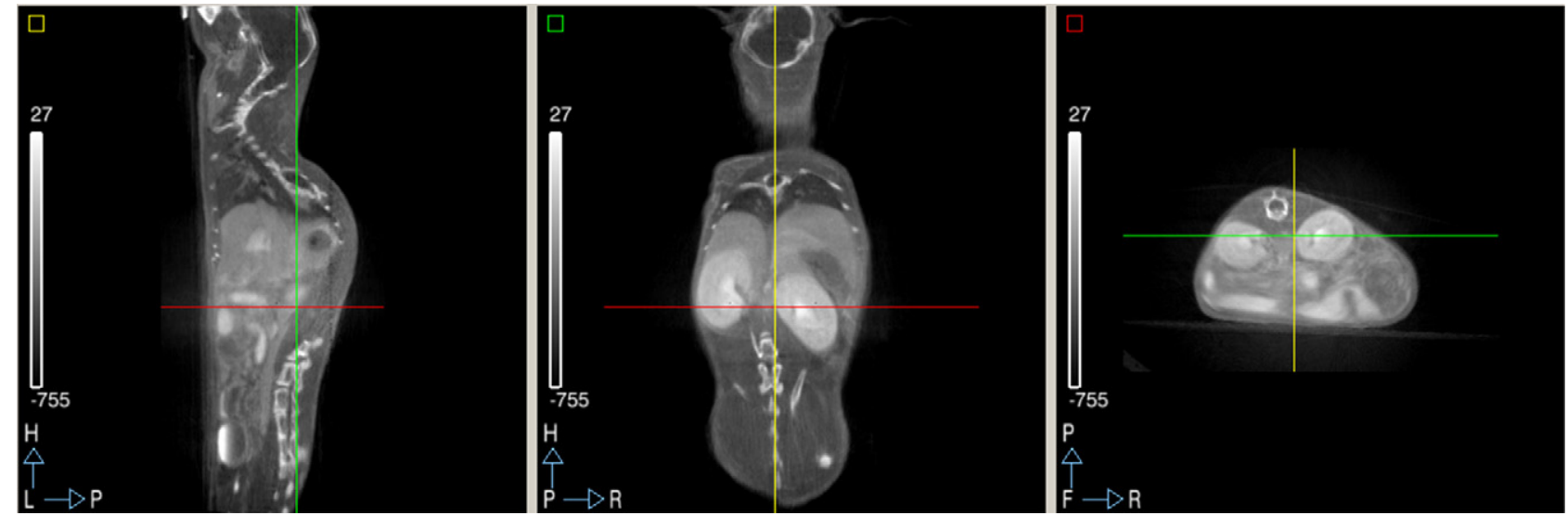

Fig. 19 - Sagittal, coronal, and axial views of a mouse abdomen with a 0.4-ml injection of Iopamiro 300 . The acquisition parameters were $35 \mathrm{kV}, 200 \mu \mathrm{A}, 360$ angular projections, $0.125 \mathrm{~mm}$ pixel size, and eight images averaged per projection (standard lab protocol for mice). Image is depicted with a soft tissue window. 


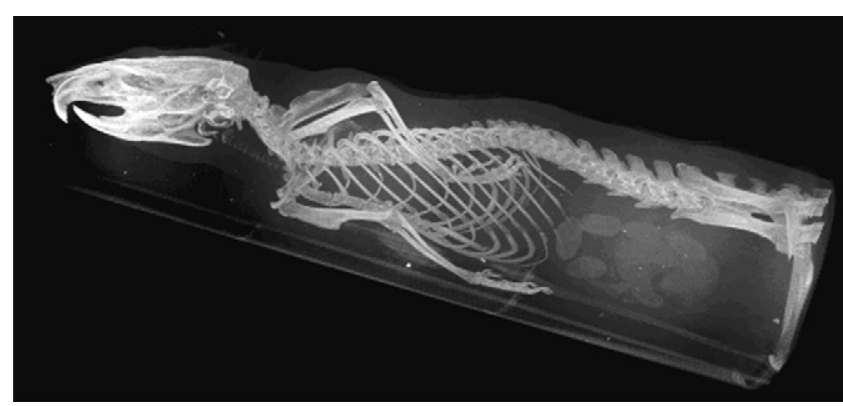

Fig. 20 - Volume render of a 3-bed rat study. The acquisition parameters were $40 \mathrm{kV}, 200 \mu \mathrm{A}, 360$ angular projections, $0.125 \mathrm{~mm}$ pixel size, and eight images averaged per projection. Image is depicted with a bone window.

architecture facilitates the substitution of any module for a GPU kernel.

The ring artifacts remaining from the flood field correction are not negligible, making it necessary to include a correction for this artifact in the workflow. For ring artifact correction, we propose an algorithm applied in the projection data before reconstruction, as it avoids the high computational burden derived from the domain transformations needed in other proposed methods, and can be efficiently included in the whole correction/reconstruction pipe-line. The strategy of dividing the sinogram into parts with a subset of angles and correct each part separately was motivated by two reasons: first, line artifacts in the sinogram are not constant along all the angles due to their dependency with the attenuation suffered along each path, thus the same correction vector would not apply for every angle; secondly, correcting each subset of projections independently avoids having to wait until all the projections are acquired to start the correction/reconstruction workflow.

We have included two algorithms for compensating beam-hardening artifacts: the linearization method that compensates cupping artifact in homogeneous phantoms is important given that such phantoms are used for HU calibration; the second-order beam-hardening correction is necessary to eliminate dark streaks between bones in rodent studies.

To obtain a good linear fitting for HU calibration at least a three material phantom covering the range of densities common to small animal experiments should be used: water-equivalent material, bone-equivalent material, and air. Phantom acquisitions should be corrected for cupping (linearization method) to assure quantitative measurements on the different materials.

No scatter correction algorithm was included; a study of scatter effect in small-animal cone-beam CT systems is out of the scope of this work. Nevertheless, given that the artifacts derived from the scatter are similar to those produced by beam-hardening and that the methods included to reduce the latter are empirical-based methods (measurements necessarily include other effects such as scatter), we expect to compensate both effects at the same time, as suggested in [53].

High-resolution imaging requires a precise characterization of the system alignment. Misalignments that could not be mechanically corrected during manufacturing have to be taken into account during the image reconstruction process. A good calibration of detector misalignments, especially linear shifts and skew of the detector, is crucial to avoid artifacts when reconstructing images of single-bed studies. At the same time, a good calibration of the magnification parameter and the mismatch of bed displacement with the rotation axis are also crucial to avoid artifacts in multi-bed studies. The magnification parameter has a direct effect on the pixel size, which affects quantification measures and the quality of image fusion with other studies of the same or different modality. Procedures with dedicated phantoms to calibrate the magnification factor and the different misalignments, within one bed and between consecutive beds, have been described. Misalignment correction in single bed studies is done on the projections as a preliminary step, while misalignment between beds is taken into account in the stitching procedure.

The main contribution of this work, besides several novelties on calibration and artifact correction, is the whole workflow proposed, since the selection criteria for the different components and their arrangement is not described elsewhere. Our results on phantoms and rodent studies show that the proposed architecture is suitable for a micro X-ray CT system based on flat-panel detectors and circular trajectory.

\section{Conflict of interest statement}

None declared.

\section{Acknowledgements}

This work was partially funded by AMIT project from the CDTI CENIT program, TEC2007-64731, TEC2008-06715C02-01, RD07/0014/2009, TRA2009_0175, RECAVA-RETIC, and RD09/0077/00087 (Ministerio de Ciencia e Inovación), and ARTEMIS S2009/DPI-1802 (Comunidad de Madrid).

\section{REFERENCES}

[1] A.L. Goertzen, V. Nagarkar, R.A. Street, M.J. Paulus, J.M. Boone, S.R. Cherry, A comparison of X-ray detectors for mouse CT imaging, Phys. Med. Biol. (2004) 5251-5265.

[2] H.K. Kim, S.C. Lee, M.H. Cho, S.Y. Lee, G. Cho, Use of a flat-panel detector for microtomography: a feasibility study for small-animal imaging, IEEE Trans. Nucl. Sci. (2005) 193-198.

[3] E.L. Ritman, Small-animal CT: its difference from, and impact on, clinical CT, Nucl. Instum. Methods Phys. Res. A (2007) 968-970.

[4] C.T. Badea, M. Drangova, D.W. Holdsworth, G.A. Johnson, In vivo small-animal imaging using micro-CT and digital subtraction angiography, Phys. Med. Biol. (2008) R319-R350.

[5] J.J. Vaquero, S. Redondo, E. Lage, M. Abella, A. Sisniega, G. Tapias, M.L.S. Montenegro, M. Desco, Assessment of a new high-performance small-animal X-ray tomograph, IEEE Trans. Nucl. Sci. (2008) 898-905.

[6] L.A. Feldkamp, L.C. Davis, J.W. Kress, Practical cone-beam algorithm, J. Opt. Soc. Am. (1984) 612-619. 
[7] G.S.L. Zeng, G.T. Gullberg, A cone-beam tomography algorithm for orthogonal circle-and-line orbit, Phys. Med. Biol. (1992) 563-577.

[8] H. Liang, C. Zhang, M. Yan, A Feldkamp-type approximate algorithm for helical multislice ct using extended scanning helix, Comput. Med. Imaging Graph. (2009) 197-204.

[9] H. Scherl, B. Keck, M. Kowarschik, J. Homegger, Fast GPU-based ct reconstruction using the common unified device architecture (CUDA), IEEE Nucl. Sci. Symp. Conf. Record (2007) 4464-4466.

[10] Y. Okitsu, F. Ino, K. Hagihara, Accelerating cone beam reconstruction using the cuda-enabled GPU, in: Proceedings of the 15th International Conference on High Performance Computing, Springer-Verlag, Bangalore, India, 2008, pp. 108-119.

[11] G.R. Yan, J. Tian, S.P. Zhu, Y.K. Dai, C.H. Qin, Fast cone-beam CT image reconstruction using GPU hardware, J. X-Ray Sci. Technol. (2008) 225-234.

[12] X. Zhao, J.-J. Hu, P. Zhang, GPU-based 3D cone-beam CT image reconstruction for large data volume, Int. J. Biomed. Imaging (2009) 149079.

[13] P.B. Noël, A.M. Walczaka, J. Xua, J.J. Corsoa, K.R. Hoffmanna, S. Schafera, GPU-based cone beam computed tomography, Comput. Methods Programs Biomed. (2010) 271-277.

[14] O. Yusuke, F. Ino, K. Hagihara, High-performance cone beam reconstruction using cuda compatible GPUS, Parallel Comput. (2010) 129-141.

[15] T. Schiwietz, S. Bose, J. Maltz, R. Westermann, A fast and high-quality cone beam reconstruction pipeline using the GPU, Proc. SPIE Med. Imaging (2007) 65105H.

[16] M. Grass, T. Kohler, R. Proksa, Angular weighted hybrid cone-beam ct reconstruction for circular trajectories, Phys. Med. Biol. (2001) 1595-1610.

[17] X.Y. Tang, J. Hsieh, A. Hagiwara, R.A. Nilsen, J.B. Thibault, E. Drapkin, A three-dimensional weighted cone beam filtered backprojection (CB-FBP) algorithm for image reconstruction in volumetric ct under a circular source trajectory, Phys. Med. Biol. (2005) 3889-3905.

[18] T. Rodet, F. Noo, M. Defrise, The cone-beam algorithm of Feldkamp, Davis, and Kress preserves oblique line integrals, Med. Phys. (2004) 1972-1975.

[19] Y. Bresler, J. Brokish, A hierarchical algorithm for fast backprojection in helical cone-beam tomography, IEEE Int. Symp. Biol. Imaging (2004) 1420-1423.

[20] F. Noo, R. Clackdoyle, C. Mennessier, T.A. White, T.J. Roney, An analytic method based on identification of ellipse parameters for scanner calibration in conebeam tomography, Phys. Med. Biol. (2000) 3489-3508.

[21] P.M. Joseph, R.D. Spital, A method for correcting bone induced artifacts in computed tomography scanners, J. Comput. Assist. Tomogr. (1978) 100-108.

[22] Y. Kyriakou, D. Prell, W.A. Kalender, Ring artifact correction for high-resolution micro CT, Phys. Med. Biol. (2009) N385-N391.

[23] A.C. Kak, Slaney, Malcolm, Principles of Computerized Tomographic Imaging, IEEE Press, 1988.

[24] T. Peters, Algorithms for fast back- and re-projection in computed tomography, IEEE Trans. Nucl. Sci. (1981) 3641-3647.

[25] I. Vidal-Migallon, M. Abella, A. Sisniega, J.J. Vaquero, M. Desco, Simulation of mechanical misalignments in a cone-beam micro-CT system, IEEE Nucl. Sci. Symp. Conf. Record (2009) 4273-4275.

[26] G.T. Gullberg, B.M.W. Tsui, C.R. Crawford, J.G. Ballard, J.T. Hagius, Estimation of geometrical parameters and collimator evaluation for cone beam tomography, Med. Phys. (1990) 264-272.
[27] A. Rougee, C. Picard, C. Ponchut, Y. Trousset, Geometrical calibration of X-ray-imaging chains for 3-dimensional reconstruction, Comput. Med. Imaging Graph. (1993) 295-300.

[28] Y. Sun, Y. Hou, F.Y. Zhao, J.H. Hu, A calibration method for misaligned scanner geometry in cone-beam computed tomography, NDT\&E Int. (2006) 499-513.

[29] Y. Cho, D.J. Moseley, J.H. Siewerdsen, D.A. Jaffray, Accurate technique for complete geometric calibration of cone-beam computed tomography systems, Med. Phys. (2005) 968-983.

[30] S.M. Johnston, G.A. Johnson, C.T. Badeaa, Geometric calibration for a dual tube/detector micro-CT system, Med. Phys. (2008) 1820-1829.

[31] L. von Smekal, M. Kachelriess, E. Stepina, W. Kalender, Geometric misalignment and calibration in cone-beam tomography, Med. Phys. (2004) 3242-3266.

[32] D. Panetta, N. Belcari, A. Del Guerra, S. Moehrs, An optimization-based method for geometrical calibration in cone-beam CT without dedicated phantoms, Phys. Med. Biol. (2008) 3841-3861.

[33] R.A. Brooks, G.D. Chiro, Beam hardening in X-ray reconstruction tomography, Phys. Med. Biol. (1976) 390-398.

[34] M. Kachelriess, K. Sourbelle, W.A. Kalender, Empirical cupping correction: a first-order raw data precorrection for cone-beam computed tomography, Med. Phys. (2006) 1269-1274.

[35] B. Li, Q. Zhang, J. Li, A novel beam hardening correction method for computed tomography, IEEE/ICME Int. Conf. Complex Med. Eng. (2007) 891-895.

[36] R. Grimmer, C. Maass, M. Kachelriess, A new method for cupping and scatter precorrection for flat detector CT, IEEE Nucl. Sci. Symp. (2009) 3517-3522.

[37] J. Hsieh, R.C. Molthen, C.A. Dawson, R.H. Johnson, An iterative approach to the beam hardening correction in cone beam CT, Med. Phys. (2000) 23-29.

[38] O. Nalcioglu, R.Y. Lou, Post-reconstruction method for beam hardening in computerised tomography, Phys. Med. Biol. (1979) 330-340.

[39] F.P. Vidal, J.M. Letang, G. Peix, P. Cloetens, Investigation of artifact sources in synchrotron microtomography via virtual X-ray imaging, Nucl. Instum. Methods Phys. Res. (2005) 333-348.

[40] J.A. Seibert, J.M. Boone, K.K. Lindfors, Flat-field correction technique for digital detectors, Phys. Med. Imaging (1998) 348-354.

[41] S. Arivazhagan, S. Deivalakshmi, K. Kannan, B.N. Gajbhiye, C. Muralidhar, S.N. Lukose, M.P. Subramanian, Multi-resolution system for artifact removal and edge enhancement in computerized tomography images, Pattern Recogn. Lett. (2007) 1769-1780.

[42] M. Axelsson, S. Svensson, G. Borgefors, Reduction of ring artifacts in high resolution X-ray microtomography images, in: Pattern Recognition, Springer, Berlin/Heidelberg, 2006, pp. 61-70.

[43] M. Boin, A. Haibel, Compensation of ring artefacts in synchrotron tomographic images, Opt. Express (2006) 12071-12075.

[44] G.H. Chen, S. Leng, A new data consistency condition for fan-beam projection data, Med. Phys. (2005) 961-967.

[45] R.A. Ketcham, in: U. Bonse (Ed.), New algorithms for ring artifact removal, Proc. SPIE (2006) 631800.

[46] C. Raven, Numerical removal of ring artifacts in microtomography, Rev. Sci. Instrum. (1998) 2978-2980.

[47] M. Rivers, Tutorial Introduction to X-Ray Computed Microtomography Data Processing, 1998.

[48] J. Sijbers, A. Postnov, Reduction of ring artifacts in high resolution micro-CT reconstructions, Phys. Med. Biol. (2004) 247-253. 
[49] E.M. Abu Anas, S.L. Lee, M.K. Kamrul Hasan, Removal of ring artifacts in ct imaging through detection and correction of stripes in the sinogram, Phys. Med. Biol. (2010) 6911-6930.

[50] F. Sadi, S.Y. Lee, M.K. Hasan, Removal of ring artifacts in computed tomographic imaging using iterative center weighted median filter, Comput. Biol. Med. (2010) 109-118.

[51] M.A. Yousuf, M. Asaduzzaman, An efficient ring artifact reduction method based on projection data for micro-CT images, J. Sci. Res. (2010) 37-45.

[52] B. De Man, S. Basu, Distance-driven projection and backprojection in three dimensions, Phys. Med. Biol. (2004) 2463-2475.

[53] E. Meyer, C. Maaß, M. Baer, R. Raupach, B. Schmidt, M. Kachelrieß, A new method for scatter correction in cone-beam CT and its application to metal artifact reduction, in: IEEE NSS/MIC Conference Record, 2010. 